\title{
LT-structural changes of the $\left(\mathrm{NbSe}_{4}\right)_{10} \mathrm{I}_{3}$ compound, a quasi-one dimensional charge density wave system
}

\author{
Z. VUČIĆ(1), A. MEETSMA and J.L. DE BOER \\ University of Groningen, Laboratory of Chemical Physics, Materials Science Center, Nyenborgh 4, \\ 9747 AG Groningen, The Netherlands
}

\begin{abstract}
In order to study the structural background of the giant thermal hysteresis observed in the thermopower measurements [1] of the CDW system ( $\left.\mathrm{NbSe}_{4}\right)_{10} \mathrm{I}_{3}$, zeroth-order Weissenberg and oscillation photographs were taken at temperatures below Peierls transition. A reversible transformation of the room temperature tetragonal lattice into four symmetricaly equivalent monoclinic domains was observed with the monoclinic angle behaving like an order parameter.
\end{abstract}

\section{Introduction}

More than ten years ago a series of new quasi-one-dimensional compounds with general formula $\left(\mathrm{MSe}_{4}\right)_{\mathrm{x}} \mathrm{I} ; \mathrm{M}=\mathrm{Ta}, \mathrm{Nb} ; \mathrm{x}=2,3,10 / 3$ has been synthesized [2,3]. At room temperatures all of them have tetragonal symmetry but different space groups (I422, P4/mnc, P4/mcc, respectively). The common structural feature is an array of parallel well separated chains of metal atoms. The chains are at positions $(0,1 / 2, \mathrm{z})$ and $(1 / 2,0, \mathrm{z})$. Each metal atom is sandwiched between two $\mathrm{Se}_{4}$ rectangular units rotated to each other by 45 degrees. There are also parallel rows of iodine atoms. The rows are at positions $(0,0, z)$ and $(1 / 2,1 / 2, z)$. Apart from the length of the $c$ axis being 4,6 and 10 metal-metal distances long for the $2 / 1,3 / 1$ and $10 / 3$ compounds, respectively, the main structural differences come from distribution of iodine atoms within rows and their numbers per unit cell. In the case of $2 / 1$ and $3 / 1$ compounds all parallel iodine rows are equivalent having 2 iodine atoms per row. In the $10 / 3$ case the rows at $(0,0, \mathrm{z})$ contain 4 atoms while the rows at $(1 / 2,1 / 2, \mathrm{z})$ contain 2 atoms thus forming the anisotropic iodine surrounding of each metal chain.

In two (2/1 and 10/3) of three compounds, CDW phenomena are observed below Peierls transition (260K and $285 \mathrm{~K}$, respectively) associated with nonlinear conductivity, switching, narrow-band noise and metastability $[3,4]$. For both CDW systems the components of $2 \mathrm{k}_{\mathrm{f}}$ distortion wave vectors are observed as predicted from the electronic band-subband consideration [5,6]. They are $( \pm 0.05, \pm 0.05, \pm 0.085)[8]$ and $(0,0, \pm 0.487)$ for $2 / 1$ and $10 / 3$ compound, respectively.

Regarding the metastability, a huge hysteresis has been recently found in thermopower vs. temperature experiments on $10 / 3$ compound [1]. In order to search for the structural background of

(1) Permanent address (from September $1^{\text {st }}$ 1993): Institute of Physics of the University, Zagreb, Bijenicka 46, P.O. Box 304, 4100 Zagreb, Republic of Croatia 
this hysteresis the single-crystal $x$-ray diffraction patterns of $10 / 3$ compound were collected using Weissenberg and oscillation methods from room temperature down to $130 \mathrm{~K}$.

\section{Experimental details}

Two single crystals of $10 \times 10 \times 20 \mu \mathrm{m}$ were selected from the same batch and glued to the goniometer head with $a$ axis aligned along the oscillation axis. The standard low-temperature Enraf-Nonius Weissenberg camera is used with $\mathrm{CuK} \alpha$ graphite-monochromated radiation and zeroth-layer $0 \mathrm{kl}$ Weissenberg and oscillation photographs were taken at different temperatures. The crystal was cooled by regulated and previously calibrated $\mathrm{LN}_{2}$ vapour stream.

\section{Results and discussion}

Weissenberg and oscillation photographs taken below Peierls transition $\left(T_{p}=280 K\right)$ have shown that Bragg spots of room temperature tetragonal phase with $l \neq 0$ are split, as a function of temperature, into 4 symmetrically equivalent spots of almost the same intensity. The indexes of the new Bragg spots in the tetragonal reference frame are: $\left(h+\eta_{l}(\mathrm{~T}), k, l\right), \quad\left(h-\eta_{l}(\mathrm{~T}), k, l\right)$, $\left(h, k+\eta_{l}(\mathrm{~T}), l\right)$ and $\left(h, k-\eta_{l}(\mathrm{~T}), l\right)$. The magnitude of splitting $\eta_{l}(\mathrm{~T})$ continuously changes from zero at $\mathrm{T}_{\mathrm{p}}=280 \mathrm{~K}$ up to $0.011 l$ at $130 \mathrm{~K}$. The splitting process is reversible with temperature. The split-

ting measured in Weissenberg photographs

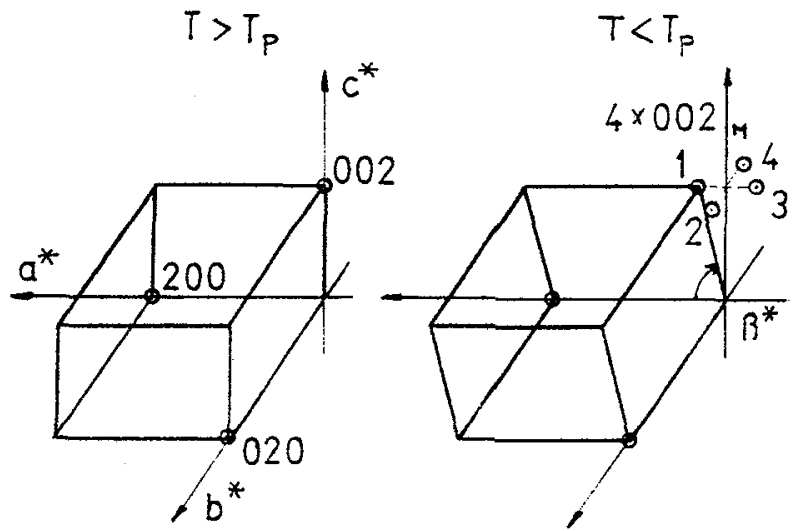

Fig.1: Schematic presentation of the Bragg splitting below Peierls transition $\left(T_{p}\right) . T>T_{p}$ : tetragonal reciprocal lattice, $T<T_{p}$ : one of four monoclinic domains reciprocal lattice. gives directly the angle between two-bytwo new $c^{*}$ axes schematically shown in Fig.1. Since we deal with the four new sets of Bragg spots instead of single tetragonal one the conversion to real space is straightforward. At $T_{p}$ there is a transformation of the tetragonal lattice into four symmetrically equivalent monoclinic lattice domains related to each other by the rotation around the fourfold axis. The monoclinic angle is a temperature dependent parameter changing from 90 degrees at $T_{p}$ up to the 92.1 degrees at $130 \mathrm{~K}$. Since there is no splitting of the spots laying in the $a^{*}, b^{*}$ plane $(l=0)$ the monoclinic deformation of the tetragonal lattice is the consequence of the relative shift of the

neighbouring chains along the chain direction, i.e. 'transverse shear mode'. More precisely, there is a shearing of neighbouring planes parallel either to the $a, c$ planes or to the $b, c$ planes. 
The temperature dependence of the monoclinic angle $\left(\beta_{M}\right)$ in the cooling-heating cycle is shown in Fig. 2. Apart from Peierls transition there are two more anomalies: one at $220 \mathrm{~K}$ and the other slightly below $200 \mathrm{~K}$. The temperature dependence of $\beta_{\mathrm{M}}$ resembles the order parameter behaviour

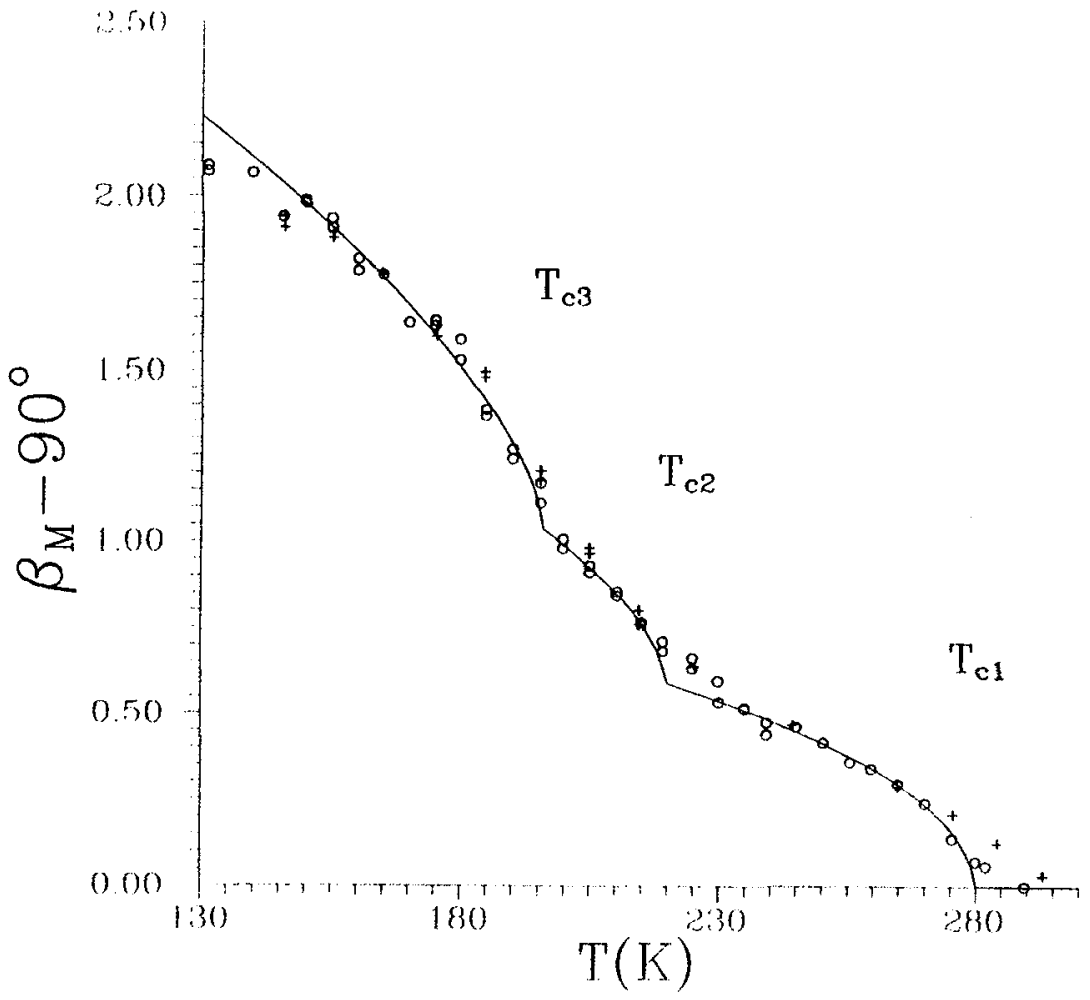

Fig.2: Variation of the monoclinic angle with temperature: cooling (o), heating ( + ). Full line is the sum of 3 critical dependences: $\beta_{M^{\alpha}}\left(T_{c i}-T\right)^{1 / 2} ; T_{c 1}=279.60 K, T_{c 2}=219.44 K, T_{c 3}=195.53 K$.

with the critical exponent being roughly $0.50 \pm 0.05$. The full line in Fig. 2 is the mean-field order parameter fit with three different independent critical temperatures keeping fixed the value of the critical exponent of $1 / 2$. In the region between $280 \mathrm{~K}$ and $220 \mathrm{~K}$ the obtained critical dependence is $\left(\beta_{M}-90\right)_{1}=0.076 \times(279.60-T)^{1 / 2}$. It is then subtracted from the measured values in the next region. The procedure is repeated in the temperature region between $220 \mathrm{~K}$ and $200 \mathrm{~K}$ and finally between $200 \mathrm{~K}$ and $130 \mathrm{~K}$. The last two steps have revealed two more relations: $\left(\beta_{\mathrm{M}^{-90}}\right)_{2}=$

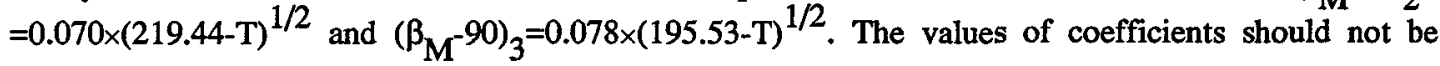
taken too strictly because of the limited precision of the measured data.

The obtained critical temperatures of $220 \mathrm{~K}$ and $195 \mathrm{~K}$ coincide with the temperatures of anomalies observed in transport measurements. Namely, $220 \mathrm{~K}$ is the temperature at which in the threshold field vs. temperature dependence the termination of the hysteresis and the change of slope is observed [4]. The other characteristic temperature $195 \mathrm{~K}$ coincides well with the maximum in the thermopower vs. temperature measurement [1]. 
Although one might notice similarity between the splitting observed in this work for $10 / 3$ compound and that observed for the $\left(\mathrm{TaSe}_{4}\right)_{2} \mathrm{I}$ compound [8] the differences seem to be more instructive. Apart from $2 \mathrm{k}_{\mathrm{f}}$ distortion wave vectors which are at different points in the Brillouine zone, i.e. near to the center and near to the edge of the zone for the $2 / 1$ and $10 / 3$ compounds, respectively, there are a few more important distinctions. First, domains observed in $2 / 1$ compound are the periodic-lattice-distortion (PLD) domains having a common Bragg tetragonal lattice. Here in the case of $10 / 3$ compound the domains are completely of Bragg type. Second, $a^{*}$ and $b^{*}$ components of the PLD wave vector in the case of $2 / 1$ compound are temperature independent. In the case of $10 / 3$ compound Bragg spot indexes $h$ or $k$ are critically dependent on temperature suggesting shear stress as an additional order parameter. Third, different splitting directions $\left( \pm a^{*} \pm b^{*}\right)$ in the case of $2 / 1$ and $\pm a^{*}$ or $\pm b^{*}$ in the case of $10 / 3$ compound) are probably due to the second-neighbours interchain interaction anisotropy, pointing to the critical importance of details of CDW interchain interactions.

It is also worth noting that at room temperature a strong diffuse scattering around all spots except those having $l=0$ is observed. Although not examined in detail, it may be described as being perpendicular to $c^{*}$ direction and extended over the whole Brillouine zone, modulated by the intensity of the parent spot. It is not clear whether it comes from a certain iodine ions disorder in the rows [2] or that its origin lays in X-ray diffraction on low-energy phonons. If the scattering on phonons is the underlying mechanism then these are TA phonons having wave vectors' directions in the $a, b$ plane and polarization vector along the chain direction. This might explain the origin of transverse shear mode in the LT-phase if a coupling to the $2 \mathrm{k}_{\mathrm{f}}$ distortion wave vector exists.

\section{Acknowledgement}

Single crystals used in this study were synthesized by Prof. F.Levy, Lausanne and supplied to us by dr. K.Biljaković, Zagreb, who also suggested the LT structural investigation. We are indebted to both of them. One of the authors, Z. Vucic wishes to thank Prof. A.Bjelis and Mr. J.Gladic for helpful discussions. This investigation was supported by Commission of the EC, DG XII, Contract number B/CI1*-913183.

\section{References}

1. A.Smontara, K.Biljaković, J.Mazuer, P.Monceau and F.Levy, J.Phys.: Condens. Matter 4 (1992) 3273.

2. A.Meerschaut, P.Gressier, L.Guemas and J.Rouxel, J. Solid State Chem., 51 (1984) 307.

3. P.Gressier, A.Meerschaut, L.Guemas, J.Rouxel and P.Monceau, J. Solid State Chem., 51 (1984) 141.

4. Z.Z.Wang, P.Monceau, M.Renard, P.Gressier, L.Guemas and A.Meerschaut, Solid State Commun., 47 (1983) 439.

5. P.Gressier, M-H.Whangbo, A.Meerschaut and J.Rouxel, Inorganic Chem., 23 (1984) 1221.

6. P.Gressier, A.Meerschaut, J.Rouxel and M-H.Whangbo, Springer Lecture Notes in Physics 217 ed G.Hutiray and J.Solyom (Berlin: Springer) 1985, p.43.

7. C.Roucau, R.Ayroles, P.Gressier and A.Meerschaut, J. Phys.C: Solid State Phys., 17 (1984) 2993.

8. H.Fujishita, M.Sato and S.Hoshino, Solid State Commun., 49 (1984) 313. 